\title{
Discriminant analysis of background noise in extremity magnetic resonance images
}

\author{
Carlos José Andrioli \\ Departamento de Engenharia Elétrica \\ Centro Universitário FEI \\ São Bernardo do Campo, Brasil \\ cmandriolis@gmail.com
}

\author{
Carlos Eduardo Thomaz \\ Departamento de Engenharia Elétrica \\ Centro Universitário FEI \\ São Bernardo do Campo, Brasil \\ cet@fei.edu.br
}

\begin{abstract}
Since the creation of the first magnetic resonance imaging (MRI) equipment in 1974, experts have been studying the continuous improvement of image quality. This work aims to study the types of background noise in images from extremity MRI system of high-field, mainly caused by Faraday Cage problems. Phantom images of 1T equipment were investigated for this study. For the acquisition of these images, a protocol called DQA (Daily Quality Assurance) was used. For this work, 45 MRI images were acquired, which were pre-classified by an expert, and analyzed by SNR, an index that quantifies the ratio between signal and image noise, and by the multivariate statistical methods PCA + MLDA. PCA served as a statistical filter, which considerably decreased the amount of input information for MLDA. When all main components were used, MLDA showed an accuracy of $93.33 \%$ and results that allowed to discriminate background noise from these images in complementarity with SNR.
\end{abstract}

\section{INTRODUÇÃO}

Desde a criação do primeiro equipamento de ressonância magnética, pelo grupo do Sir. Peter Mansfield em 1974 na Universidade de Notthingham [McRobbie et al. 2006], que foi reconhecido pelo Comitê Nobel em 2003, devido a sua inédita concepção de utilizar campo magnético e ondas de rádio para obtenção de imagens de órgãos do corpo humano; cientistas, médicos e especialistas trabalham constantemente no aperfeiçoamento da qualidade de aquisição e processamento dessas imagens [Constantinides et al. 1997]; [Kuperman 2000]; [Jin et al. 2015].

A qualidade da imagem é medida principalmente pela relação entre o sinal emitido e a quantidade de ruído obtida na imagem, chamada de $S N R$. O valor da $S N R$ decorre de vários fatores que influenciam diretamente no sinal e no ruído da imagem, como intensidade de campo magnético, situação da homogeneização do campo, tipo de bobina de recepção, eficiência da gaiola de Faraday, interferências eletromagnéticas e temperatura.

Os primeiros trabalhos de cálculo da $S N R$ foram publicados na década de 1970 [McRobbie et al. 2006], mas foi a partir de 1990, que trabalhos mais predominantes começaram a ser publicados por [Marcovski 1996]; [Constantinides et al. 1997]; [Goerner and Clarke 2011]; [Guerin et al. 2017]. Basicamente, todos esses trabalhos estão relacionados com a definição da $S N R$, do comportamento da $S N R$ em relação à intensidade de campo magnético, da relação ao tipo de bobina utilizada e da SNR em paralelismo de imagens (pós-processamento das imagens). A maioria dos autores [Constantinides et al. 1997]; [Jin et al. 2015]; [Kuperman 2000] utilizaram em seus estudos, ressonâncias com as mais variadas intensidades de campo magnético, e por mais que existam outros procedimentos para a qualificação da qualidade de imagem, a $S N R$ é a principal e a mais utilizada nos equipamentos de RM. O processo de medida da $S N R$ é realizado por meio da análise de amostras do fundo da imagem e da imagem principal. Porém, esse processo não é aconselhável para o caso de paralelismo de imagens (pós/ processamento) [Guerin et al. 2017], visto que, nesse caso, o fundo da imagem também será processado e perderá a referência. Há várias técnicas e métodos adotados para a análise multivariada de dados de alta dimensão, entre esses pode-se citar o PCA, SVM, LDA e MLDA. [Giraldi et al. 2008]; [Sato et al. 2009]; [Fisher 1936]; [Koriwakova et al. 2010]; [Pang and Mak 2015]; [Janousova et al. 2015]; [Sharma 2017] e [Hastie et al. 2009]. Para estudos de imagens a análise discriminante realizada através do $P C A+M L D A$ é amplamente utilizada.

Dessa forma, o trabalho tem por objetivo realizar a análise de imagens de ressonância magnética, e em especial das imagens de Phantom e seus ruídos através da $S N R$ e da análise discriminante $(P C A+M L D A)$, e verificar o comportamento dos dois processos.

\section{Materiais e Métodos}

Seguem os materiais e métodos utilizados para a elaboração do trabalho.

\section{A. Equipamento}

Utilizou-se equipamentos de ressonância magnética nuclear de extremidades, de alto campo magnético, de intensidade de 1 Tesla, marca ONI, modelo MSK Extreme.

\section{B. Phantom}

É utilizado para a aquisição de imagens, trata-se de uma garrafa de material plástico contendo água desmineralizada somada de sulfato em conhecida proporção. Ao ser aplicada a essa solução um campo magnético, e em seguida pulsos de rádio frequência, a mesma, responderá ao sistema de ressonância magnética com uma certa quantidade de energia, 
que será captada pela bobina de rádio frequência do equipamento. E, por ser conhecida a concentração da solução de dentro do Phantom, pode-se estimar a resposta do Phantom, e assim determinar se o equipamento encontra-se dentro das características corretas para seu funcionamento. Na Figura 1, são apresentados alguns tipos de Phantoms utilizados para calibração e verificação de qualidade de imagem.
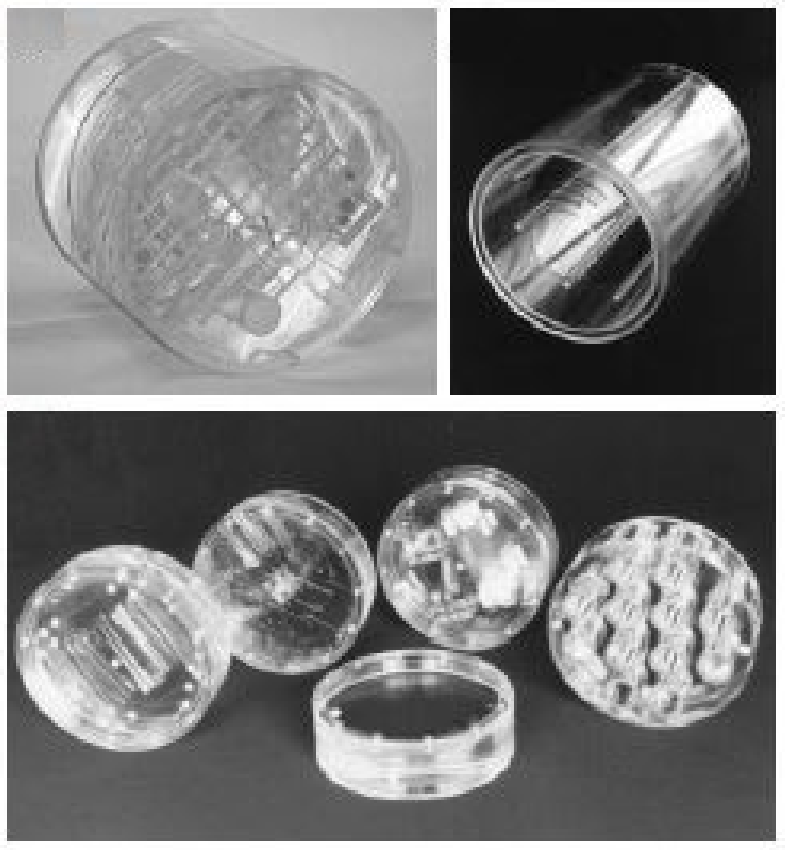

Fig. 1. Exemplos de Phantom em RM

\section{Imagens}

As imagens, resultantes das aquisições, tem aspectos muito parecidos e correspondem à uma figura circular na região central, que representa a área de um corte axial realizado no Phantom. Um quadrado, na parte interna da figura circular da região central, indica a área de aquisição do sinal da imagem chamado IS (Intensity Signal). Quatro retângulos, localizados em volta da figura central da imagem e dentro do fundo preto, sendo que, dois, situados à direita e à esquerda da figura central, representam a área de onde foram retirados os dados referentes ao ruído das imagens, e dois, situados na parte superior e inferior da figura central, representam a área de onde foram retirados os dados de uma possível vibração no magneto, que pode ser devido a bobina de gradiente mal fixada, ou vibração estrutural de onde o magneto está instalado. A Figura 2 ilustra como é realizada a retirada das amostras das imagens e a Figura 3 ilustra uma imagem de Phantom padrão.

\section{Protocolo de Aquisição}

DQA (Daily Quality Assurance) [Oni 2003] é um protocolo utilizado para aquisição e análise de imagens, utilizado para verificar as condições técnicas e confirmar se o equipamento

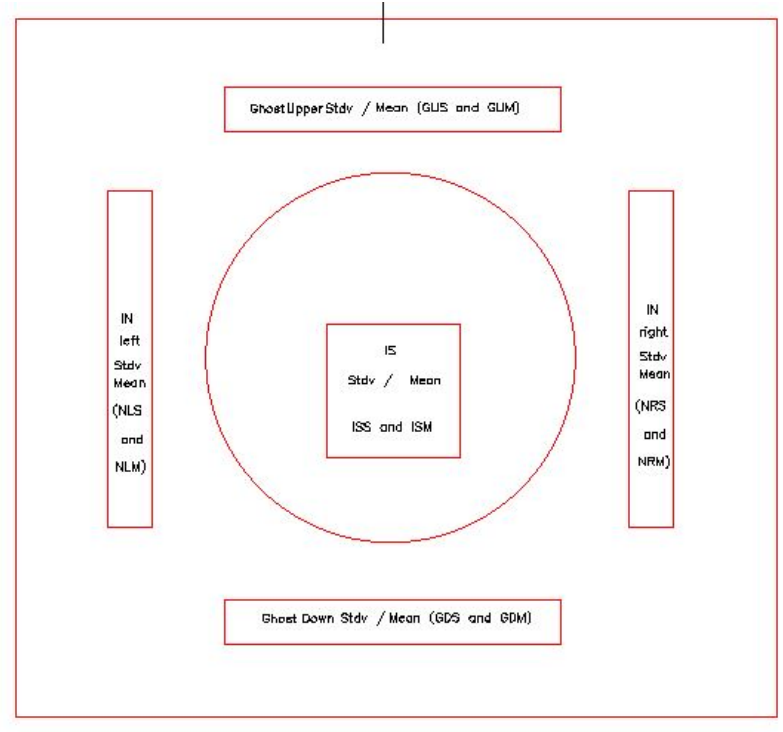

Fig. 2. Análise da imagem de Phantom

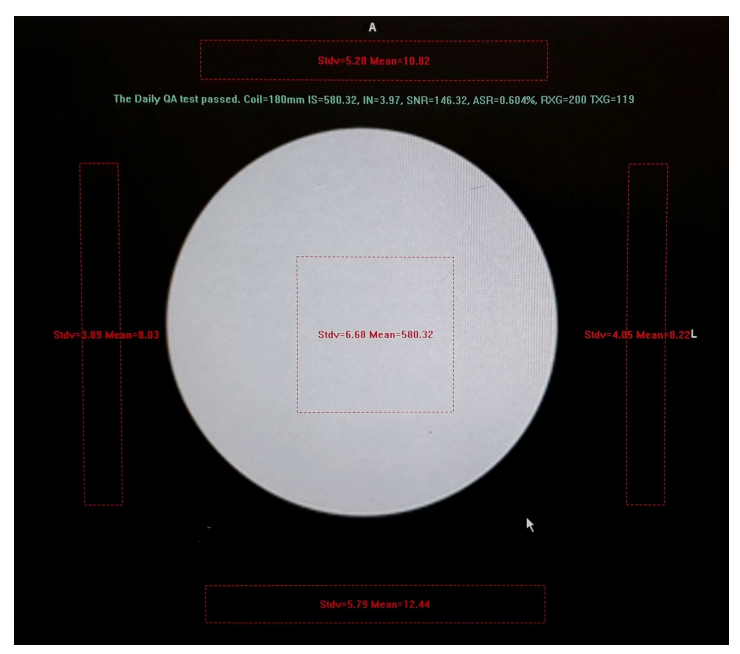

Fig. 3. Imagem de Phantom

está calibrado, e apto para a realização de exames e que foi o padrão utilizado na realização do trabalho, conforme Figura 4. O DQA define os seguintes parâmetros: tempo de aquisição de 4:19 minutos; 256 linhas x 256 colunas de aquisição; relação do FOV (Field of Vision) de 1 (número de linhas iguais ao número de colunas); espessura do corte em $5 \mathrm{~mm}$; FOV de $140 \mathrm{~mm}$; Nex igual a 1 (quantidade de vezes que um voxel será coletado e analisado); tempo de relaxamento de 1000 ms (tempo de retorno do Spin à posição inicial); tempo de excitação de $20 \mathrm{~ms}$; tempo de excitação do spin com RF) e banda passante de $25 \mathrm{kHz}$. E, em conjunto com o DQA, foi utilizada uma bobina de $180 \mathrm{~mm}$ de diâmetro, porque o tamanho da bobina interfere diretamente na relação da $S N R$.

\section{E. Definição do número de imagens}

Através do protocolo $D Q A$ foram coletadas 45 imagens de 3 equipamentos de ressonância de extremidades ONI de 1.0T 


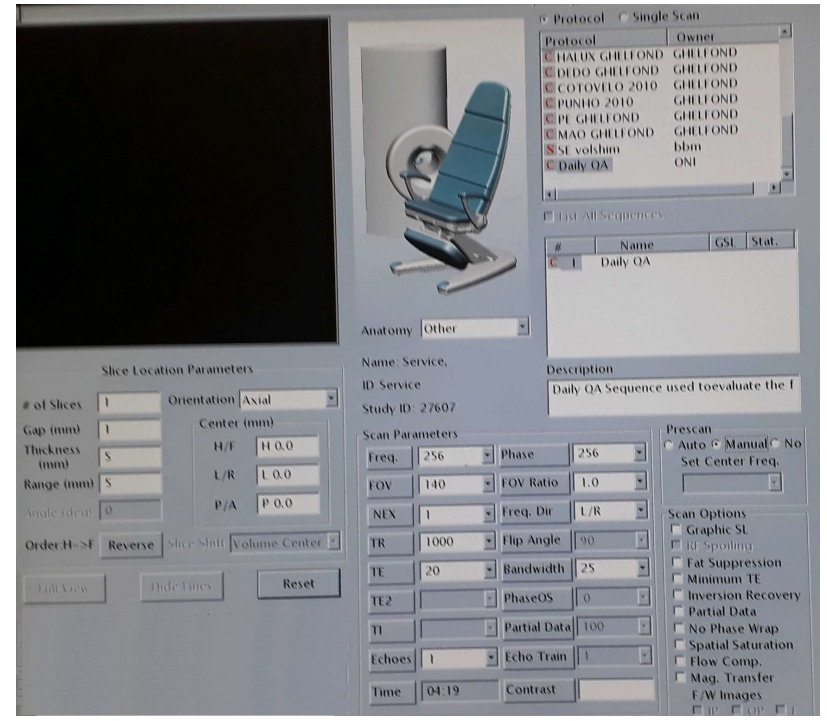

Fig. 4. Protocolo DQA

instalados em locais diferentes. As imagens foram classificadas em boas e ruins por um especialista em equipamentos de ressonância magnética considerando os seguintes critérios: apresentar geometria característica da imagem de Phantom; não ter artefatos; não apresentar ruído na imagem e ter homogeneidade na área que representa o Phantom. Nas Figuras 5 e 6 estão as imagens estudadas.

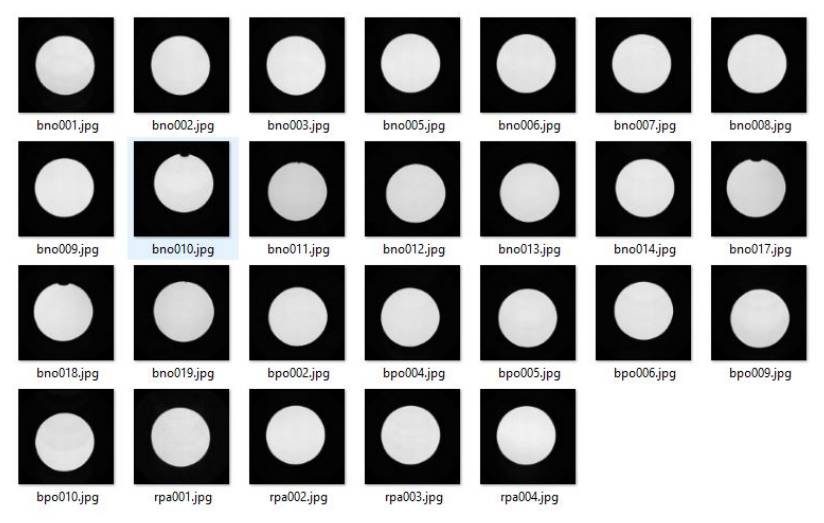

Fig. 5. Imagens Boas

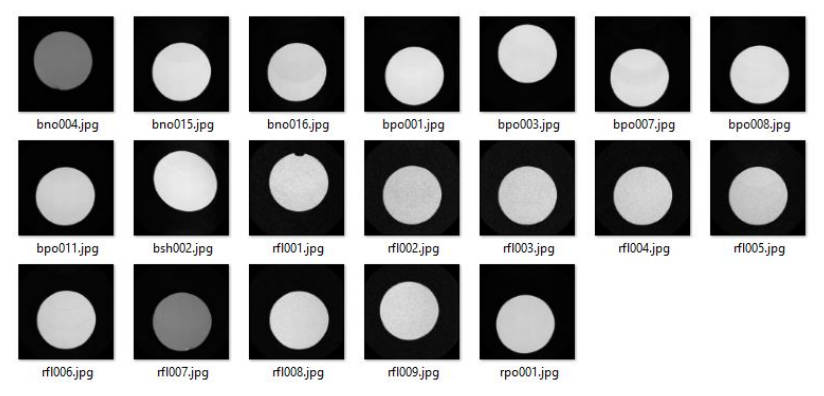

Fig. 6. Imagens Ruins

\section{F. Processo de medida da SNR pelo equipamento}

Através do protocolo $D Q A$, o equipamento automaticamente realiza os cálculos para verificar se as imagens estão dentro ou fora do padrão de qualidade, especificado pelo fabricante. Para uma bobina de $180 \mathrm{~mm}$ o $D Q A$ deve apresentar uma $S N R$ acima de 105 .

\section{G. Análise Estatística Multivariada (PCA + MLDA)}

Cada imagem foi convertida em pontos (ou amostras) no espaço n-dimensional. Como cada imagem tem 256 linhas $\mathrm{x}$ 256 colunas de resolução, o número de variáveis $n$ será de 65.536. De acordo com [Fukunaga 1990], problemas podem surgir com pequenas amostras e muitas variáveis, conhecido como problemas de poucas amostras. Há várias técnicas e métodos adotados para a análise multivariada de dados de alta dimensão, entre esses pode-se citar o PCA, SVM, LDA e $M L D A$. [Giraldi et al. 2008]; [Sato et al. 2009]; [Fisher 1936] e [Hastie et al. 2009]. O trabalho abordou os métodos PCA e $M L D A$.

\section{H. Processo de Análise dos Componentes Principais (PCA)}

A metodologia PCA (Principal Component Analysis) pode ser entendida como uma forma de aprendizado não supervisionado, que recebe como entrada uma base de dados sem nenhuma referência ou critério de agrupamento, e maximiza no espaço original, as direções de acordo com as maiores variâncias dos dados. Geometricamente, o PCA pode ser entendido como uma transformação linear do sistema de coordenadas, a partir de uma base de dados original, para um novo sistema de coordenadas rotacionado em relação ao original. Este novo sistema de coordenadas é composto por vetores ortogonais entre si, mostrando as direções em que ocorrem as maiores variâncias da base de dados original [Thomaz et al. 2006], [Fukunaga 1990].

Após aplicar o PCA (considerando as 44 componentes principais válidas), as informações se resumem a 45 imagens de 44 componentes, totalizando 1980 informações. O PCA serviu como um filtro estatístico de informações mais expressivas.

A técnica MLDA (Maximum uncertainty Linear Discriminant Analysis) foi elaborada por [Thomaz and Gillies 2006], para resolver o problema de análise de amostras de pequenas quantidades. A técnica consiste em substituir a matriz de espalhamento $S_{w}$ por outra regularizada $S_{w}^{*}$, aumentando o espalhamento e mantendo as variações mais relevantes nas amostras [Thomaz and Gillies 2006].

\section{Resultados}

Os resultados, das imagens coletadas dos equipamentos de ressonância magnética de extremidades ONI de 1.0T, em três instalações diferentes, são apresentados a seguir.

\section{A. Variância e Discriminância}

A comparação entre a Variância representada pelo resultado do $P C A$ e a Discriminância pelo $M L D A$ é exemplificada na Figura 7, que observa-se, que a variância diminui exponencialmente em relação ao índice das características principais. 
A discriminância não apresenta decréscimo em função das mesmas características.

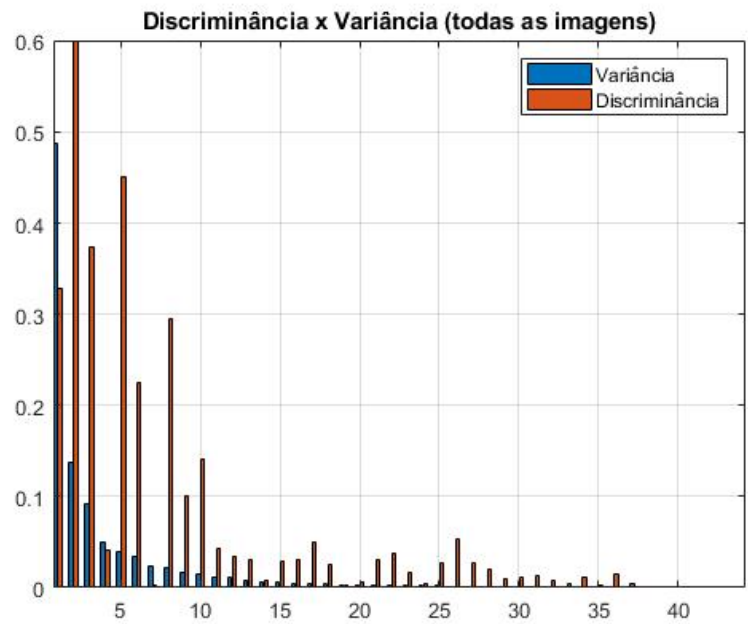

Fig. 7. Gráfico da Variância e Discriminância para todas as imagens

Observou-se que, o processo de $M L D A$, utilizou todas as componentes principais do $P C A$ e separou, total e satisfatoriamente, as imagens boas e ruins, conforme mostra o gráfico da Figura 8. Ocorreu a separação correta de 25 das 26 imagens boas analisadas, e 18 do total de 19 imagens ruins.

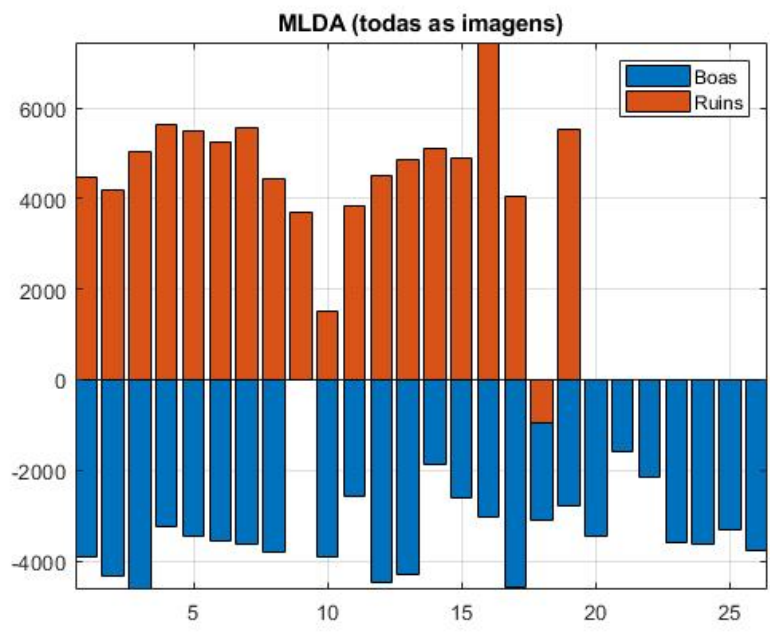

Fig. 8. Gráfico $M L D A$ para todas as principais componentes

Pela Figura 9, verifica-se a separação entre imagens boas e ruins de acordo com os processos SNR e MLDA.

Seguem as explicações das classificações das imagens analisadas:

Caso 1) São 8 imagens consideradas ruins pelo especialista e pelo $M L D A$, mas que pela $S N R$ calculcada automáticamente pelo equipamento, são consideradas boas. As imagens apresentam erros de posicionamento, artefatos, geometrias erradas e demais problemas de imagens que podem ser observadas na Figura 10.

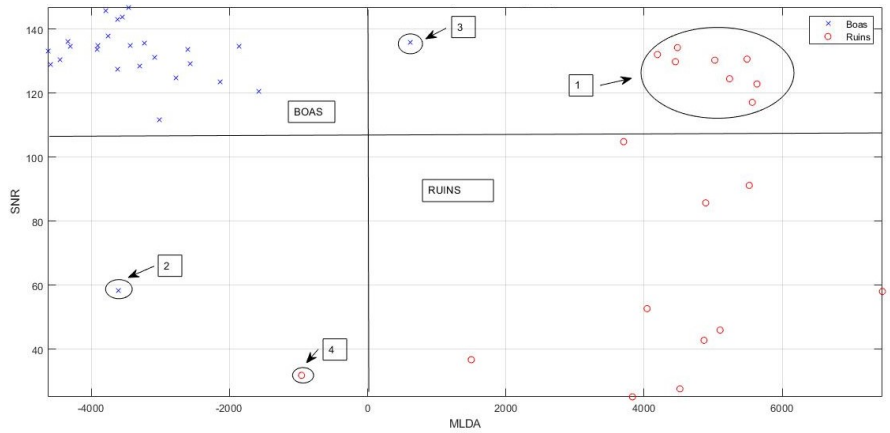

Fig. 9. Detalhe do gráfico $S N R$ e $M L D A$ das Imagens Boas e Ruins

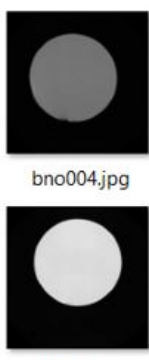

bpo003.jpg

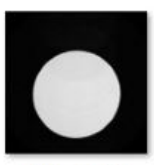

bno015.jpg

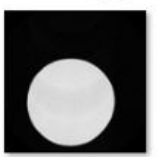

bpo007.jpg

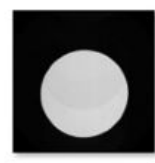

bno016.jpg

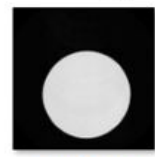

bpo008.jpg

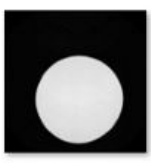

bpo001.jpg

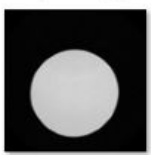

bpo011.jpg
Fig. 10. (Caso 1) Imagens boas pela $S N R$ e ruins pelo especialista e $M L D A$

Caso 2) A imagem é considerada pelo especialista e pelo $M L D A$ como boa, por não apresentar erro de posicionamento, artefato, geometria errada e demais problemas de imagem, e que pela $S N R$ foi considerada como ruim, por apresentar valor próximo a 60 (menor que 105) conforme apresentada na Figura 11.

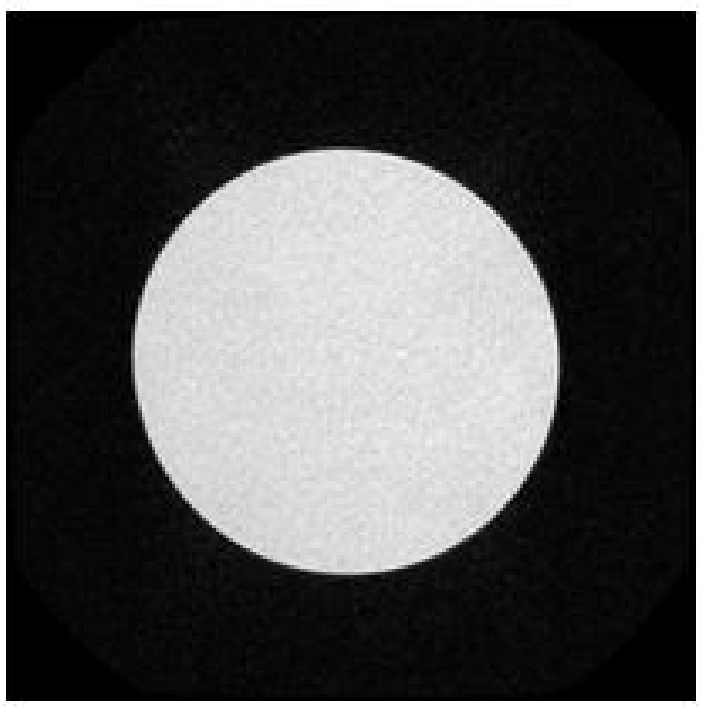

Fig. 11. (Caso 2) Imagem ruim pela $S N R$ mas boa pelo especialista e $M L D A$

Caso 3) A imagem é considerada boa pelo epecialista e 
pela $S N R$, por apresentar um fator maior que 105 , mas ruim pelo $M L D A$. Nota-se que na imagem o posicionamento está levemente acima do centro e que há uma falha na borda do Phantom (falta de líquido no Phantom - presença de ar), conforme ilustrado na Figura 12.

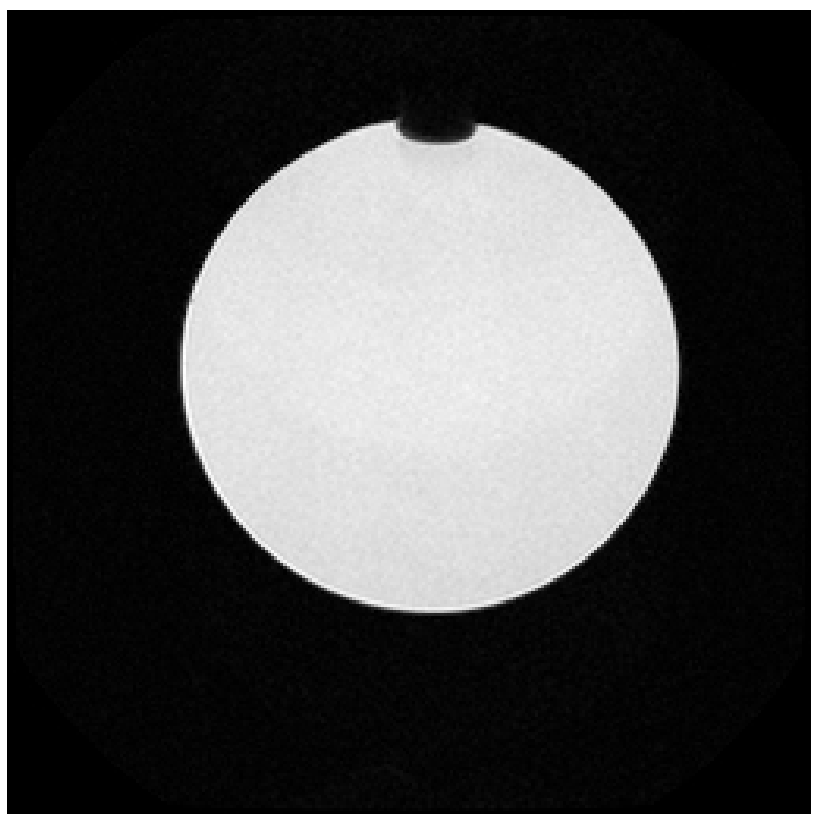

Fig. 12. (Caso 3) Imagem boa pela $S N R$ e especialista, mas ruim pelo $M L D A$

Caso 4) A imagem é considerada ruim pela $S N R$ e pelo especialista, e boa pelo MLDA. A imagem apresentou uma granulação acima do normal, não aprovada pelo especista e SNR abaixo do mínimo para ser considerada boa. Por ser homogênea por toda a área, centralizada e sem artefatos de borda, o processo de MLDA a considerou boa, conforme ilustada na Figura 13.

\section{B. Processo Leave one out}

A confiabilidade do sistema foi testada e confirmada pelo teste de leave one out, que consiste em retirar uma das amostras de classificação conhecida, realizar a classificação sem a amostra retirada e, posteriormente, calcular separadamente a amostra retirada em relação ao sistema, verificando se a mesma encontra-se boa ou ruim.

A Figura 14 representa o processo de leave one out aplicado, sendo:

1) $X=$ Imagens boas e ruins;

2) $P=$ Valores da Variância;

3) $Y=$ Classificação das imagens com os valores da variância aplicados;

4) $L=$ Valores da Discriminância;

5) $Z$ = Classificação das imagens com os valores da discriminância aplicados;

6) $B=$ Imagem única selecionada;

7) $C=$ Classificação da imagem selecionada com os valores da variância aplicados;

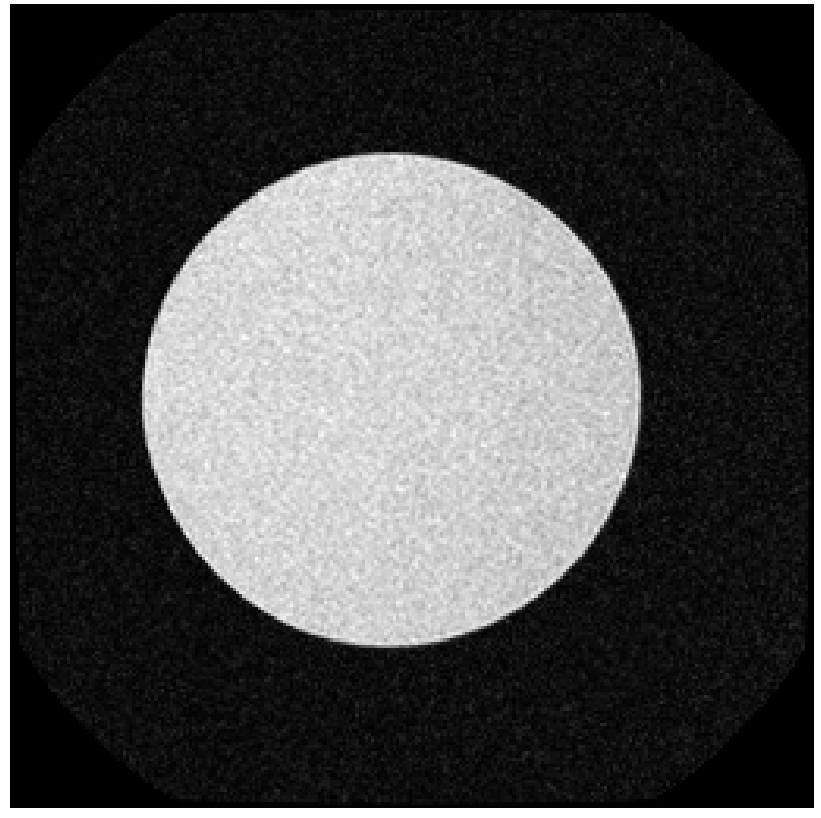

Fig. 13. (Caso 4) Imagem ruim pela $S N R$ e especialista mas boa pelo $M L D A$

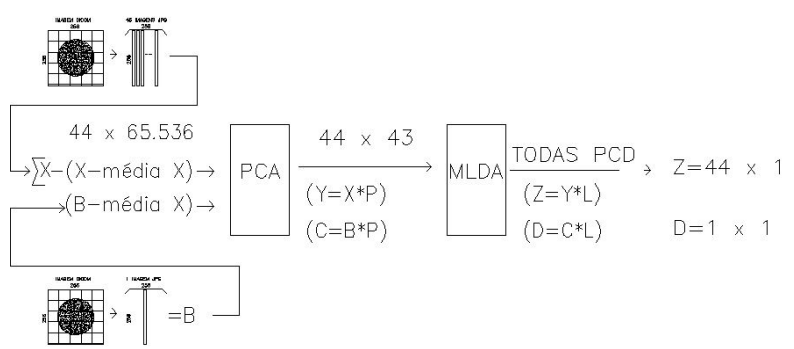

Fig. 14. Processo Leave one out

8) $D=$ Classificação da imagem selecionada com os valores da discriminância aplicados;

Para cada amostra analisada, verifica-se o valor de D, e se esse está mais próximos dos valores das imagens boas ou ruins. Como é conhecida a condição da imagem B (se ela é boa ou ruim), verifica-se a resposta do sistema referente a análise da única imagem separada $\mathrm{D}$, e confirma-se se o processo acertou ou errou na análise (distância de D para o restante das amostras). As respostas para cada amostra realizada encontrase na Figura 15.

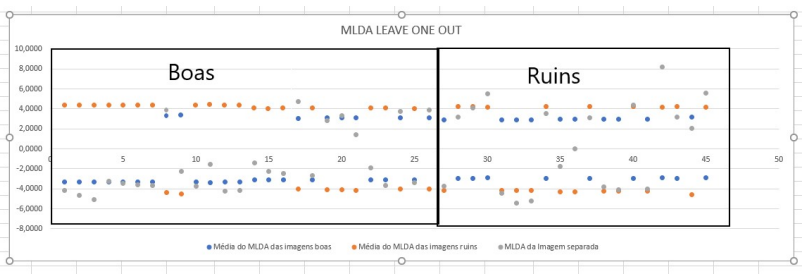

Fig. 15. Gráfico Leave one out 


\section{Acurácia}

Trata-se da proporção de resultados corretos que o classificador alcançou, ou seja, a razão entre as predições corretas pelo total. Como resposta o trabalho apresentou acerto de 42 imagens das 45 analisadas, representando uma acurácia de 93,33\%

Um exemplo de classificação de imagem utilizado no trabalho, é dado na Figura 16. A imagem verificada separadamente (rpa004) foi classificada pelo especialista e pelo código (classificador) como boa, representada no código de barras em laranja.

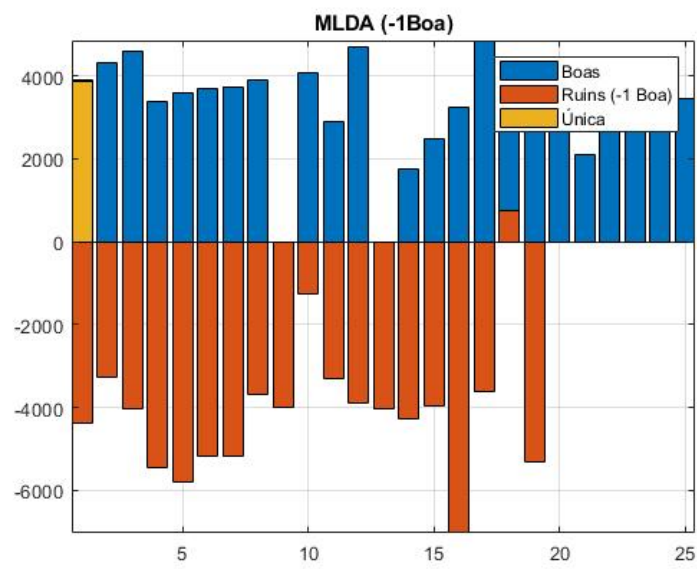

Fig. 16. Gráfico do MLDA para uma amostra boa

\section{CONCLUSÃO}

Conclui-se que vários fatores que atuam na qualidade da imagem de ressonância passam desapercebidos pela $S N R$, mas são considerados durante a Análise Estatística Multivariada $(P C A+M L D A)$, como a geometria da imagem, o posicionamento, a borda, o artefato, entre outros.

$\mathrm{O}$ processo apresentou a eficiência e acurácia informada se e somente se, for aplicado o PCA e em seguida o MLDA. O $P C A$ atuou como um filtro estatístico, diminuindo a quantidade de informação a ser calculada pelo $M L D A$.

Como verificado, o uso do (PCA + MLDA) e SNR garantem uma confiabilidade muito maior na avaliação da qualidade de imagem. Todos os equipamentos deveriam incorporar ao seu software, o código de $(P C A+M L D A)$ para a análise da qualidade da imagem, permitindo que o equipamento realize exames, apenas após ser aprovado pelos dois processos de avalição da qualidade de imagem, a $S N R$ e o (PCA + MLDA).

A base utilizada para o estudo foi de 45 imagens, e os resultados obtidos foram extremamente satisfatórios.

O trabalho limitou-se a estudar ruídos de rádio frequência e interferência eletromagnética e realizar a análise de imagens em equipamentos de ressonância magnética de extremidades de 1.0T (campo alto), pelo fato de se conhecer o especialista nesse tipo de equipamento, mas poderá ser aplicado em outros equipamentos radiológicos de imagem, e em imagens patológicas, desde que acompanhado sempre por um especialista.
A base de dados atual encontra-se disponível de forma aberta para novos trabalhos no site www.overleaf.com/project/615f8979ad722d8falc76fcc, e como sugestão, propõe-se a análise dos ruídos ocasionados por outros fatores, como interferência ferromagnética, vibração, temperatura da sala, entre outros.

\section{REFERENCES}

[Constantinides et al. 1997] Constantinides, C., Atalar, E., and McVeigh, E. R. (1997). Signal-to-noise measurements in magnitude images from nmr phased arrays. Magnetic Ressonance in Medicine, 36:852-857.

[Fisher 1936] Fisher, R. A. (1936). The use of multiple measurements in taxonimic problems. Annals of Eugenics, 7(2):179-188.

[Fukunaga 1990] Fukunaga, K. (1990). Introduction to Statistical Pattern Recognition. Cambridge University Press.

[Giraldi et al. 2008] Giraldi, G., Thomaz, C., and Rodrigues, P. (2008). Dimensionality reduction, classification and recosnstruction problems in statistical learning approaches. Revista de Informatica Teórica e Aplicada.

[Goerner and Clarke 2011] Goerner, F. L. and Clarke, G. D. (2011). Measuring signal to noise ratio in partially paralell imaging mri. Medical Physics, 38:5049-5057.

[Guerin et al. 2017] Guerin, B., Villena, J. F., Polimeridis, A. G., Adalsteinsson, E., Daniel, L., White, J. K., and Wald, L. L. (2017). The ultimate signal-to-noise ratio in realistic body models. Magnetic Ressonance in Medicine, 78:1969-1980.

[Hastie et al. 2009] Hastie, T., Tibshirani, R., and Friedman, J. (2009). The Elements of Statistical Learning:Data Mining, Inference and Prediction. Springer.

[Janousova et al. 2015] Janousova, E., Schwarz, D., and Kasparek, T. (2015) Combining various types of classifiers and features extracted from magnetic resonance imaging data in schizophrenia recognition. Psychiatry Research: Neuroimaging, 232:237-249.

[Jin et al. 2015] Jin, N. r., Saybasili, H., and Bi, X. (2015). Sparse coding for improved signal-to-noise ratio in mri. Basic Principles of Cardiovascular MRI, pages $41-62$.

[Koriwakova et al. 2010] Koriwakova, E., Schwarz, D., and Kaparek, T. (2010). Classification of 3-d mri brain data using modified maximum uncertainty linear discriminant analysis.

[Kuperman 2000] Kuperman, V. (2000). Magnetic Ressonance Imaging. Academic Press.

[Marcovski 1996] Marcovski, A. (1996). Noise in mri. Magnetic Ressonance in Medicine, 36:494-497.

[McRobbie et al. 2006] McRobbie, D., Moore, E., Graves, M., and Prince, M. (2006). Mri from picture to proton. Academic Press.

[Oni 2003] Oni (2003). Optimizing image quality.

[Pang and Mak 2015] Pang, X. and Mak, M.-W. (2015). Noise robust speaker verification via the fusion of snr-independent and snr-dependent plda. International Journal of Speech Technology, 18:633-648.

[Sato et al. 2009] Sato, J. R., Fujita, A., Thomaz, C. E.and Martin, M. G. M., Mourão, M. J., Brammer, M. J., and Junior, E. A. (2009). Evaluating svm and mlda in the extraction of discriminant regions for mental state prediction. Neuroimage, 46(1):105-114.

[Sharma 2017] Sharma, N. (2017). Single-trial p300 classification using pca with lda, qda and neural networks. ArXiv, abs/1712.01977.

[Thomaz and Gillies 2006] Thomaz, C.E.and Kitani, C. and Gillies, D. (2006). A maximum uncertainty lda-based approach for limited sample size problems - with application to face recognition. Journal of the Brazilian Computer Society (JBCS), 12(2):7-18.

[Thomaz et al. 2006] Thomaz, C.E.and Kitani, E., P.S., R., Girald, G., Rueckert, D., and Gillies, D. (2006). Discriminant principal components. Journal of the Brazilian Computer Society (JBCS), 1(2):1-12. 